\title{
First tentative detection of anisotropy in the QSO distribution around nearby edge-on spiral galaxies
}

\author{
M. López-Corredoira ${ }^{1}$ and C. M. Gutiérrez ${ }^{1}$
} Instituto de Astrofísica de Canarias, C/.Vía Láctea, s/n, 38200 La Laguna (S/C de Tenerife), Spain
e-mail: martinlc@iac.es

Received 16 March 2006 / Accepted 13 September 2006

\section{ABSTRACT}

\begin{abstract}
Aims. To check whether the polar angle distribution of QSOs around nearby spiral galaxies is isotropic or not. Methods. A statistical analysis of the polar angle distribution of large samples of QSOs from the SDSS survey and Monte Carlo simulations to calculate their significance are carried out.

Results. There is a clear excess of QSOs near the minor axis with respect to the major axis of nearby edge-on spiral galaxies, significant at a level $3.5 \sigma$ up to angular distances of $\sim 3^{\circ}$ (or $\sim 1.7 \mathrm{Mpc}$ ) from the centre of each galaxy. The significance is increased to $3.9 \sigma$ with the $z>0.5$ QSOs, and it reaches $4.8 \sigma$ if we include galaxies whose circles of radius 3 degrees are covered by the SDSS in more than $98 \%$ (instead of $100 \%$ ) of the area.

Conclusions. Gravitational lensing in the halo of nearby galaxies or extinction seem insufficient to explain the observed anisotropic distribution of QSOs. The anisotropic distribution agrees qualitatively with the predictions of Arp's models, which claim that QSOs are ejected by galaxies along the rotation axis, although Arp's prediction give a distance of the QSOs $\sim 3$ times smaller than that found here. In any case, a chance fluctuation, although highly improbable, might be a possibility rather than a true anisotropy, and the present results should be corroborated by other groups and samples, so we prefer to consider it as just a first tentative detection.
\end{abstract}

Key words. quasars: general - galaxies: statistics - catalogs - distance scale - gravitational lensing

\section{Introduction}

The first hints of a possible relationship between nearby galaxies and high redshift QSOs came from Arp $(1966,1967)$, who observed radio sources across active galaxies that were identified as QSOs. Since the pioneering work by Burbidge et al. (1971), several groups have demonstrated the existence of an angular correlation between samples of QSOs and low redshift galaxies (e.g. the reviews of Guimaraes 2005 and Burbidge 2001 respectively of orthodox and heterodox approaches). This correlation extends to angular scales of $\sim 1$ degree. Although weak gravitational lensing by dark matter has been proposed to be the cause of these correlations, many authors have found this hypothesis insufficient to explain the correlations (Benítez et al. 2001; Gaztañaga 2003; Nollenberg \& Williams 2005). The recent analysis carried out by Scranton et al. (2005), who used Sloan Digital Sky Survey (SDSS) photometric data containing $\sim 2 \times 10^{5}$ quasars and $\sim 1.3 \times 10^{6}$ galaxies, showed that the amplitude and sign of the angular correlation function between both sets of objects depend on the magnitude limit considered for the sample of QSOs. From this correlation, Scranton et al. (2005) proposed an ad hoc halo distribution function compatible with a cross-correlation of very small amplitude $\left(\omega_{\mathrm{GQ}}<0.04\right)$ of faint galaxies with QSO candidates selected photometrically $(5 \%$ of this sample are not QSOs; Richards et al. 2004). It is small because the mean separation among galaxies is small and any positive correlation of QSOs around a galaxy is diluted with the contamination of many other QSOs belonging to other galaxies. This still seems insufficient to solve the most important problem of the correlation found between QSOs and the nearest and brightest galaxies (Kovner 1989); for instance, for the high amplitude angular correlation found by Chu et al. (1984) $\omega_{\mathrm{GQ}} \sim$ 5. Although incompleteness could be responsible for these correlations, it is a matter that requires further study.

The existence of a correlation between samples of objects with different redshifts has been advocated by the supporters of non-cosmological redshifts (e.g. Burbidge 1999) as clear evidence of physical association and as proof that QSOs are being ejected by low redshift galaxies. One variant of the model (e.g. Arp 1998a, Chap. 3; Arp 1999a) assumes that QSOs are ejected along the rotation axis of the parent galaxy, decreasing in redshift as they move outwards and eventually becoming normal galaxies.

There have been claims of anisotropy in the distribution of QSOs around nearby galaxies. For instance, there are configurations of QSOs aligned along the minor axis of a central Seyfert whose probability of being accidental is $10^{-9}$ to $10^{-10}$ (Arp 1998b, 1999a). Arp \& Hazard (1980) have reported three QSOs in a straight line, together with another set of three QSOs in a straight line, a configuration that is very difficult to explain as random. Arp (1999b) finds that the probability of having six out of six QSOs aligned within $\pm 15^{\circ}$ of the minor axis of NGC 5985 to be only $10^{-8}$ to $10^{-9}$. Arp \& Russell (2001) found that the bright radio quasars $3 \mathrm{C} 37$ and $3 \mathrm{C} 39$ are paired across the centroid of the disturbed galaxy pair NGC 470/474 in an arrangement with a probability of only $2 \times 10^{-9}$ of being accidental. There is anisotropy in the radio QSO distribution at high flux densities (Shastri \& Gopal 1983): the number of QSOs on one side of the M 33 region is far greater $(\sim 11 \sigma)$ than that of the diametrically opposite region. This alignment of QSOs with the parent galaxy would involve conservation of momentum in 
their ejection in the heterodox Arp/Burbidge/Narlikar scenario (Narlikar \& Das 1980).

In this paper we undertake the first study of the polar angle distribution of QSOs around bright nearby galaxies in statistical terms using large samples of both types of objects. With the release of the SDSS survey, a systematic search for this effect is now possible. The motivation of this research is to test one of the predictions given by the above authors concerning the ejection model. The predictions are as follows: if the QSOs were background objects, no preferential orientation in principle should be found; however, if the QSOs were ejected by the galaxies, as Arp proposed (or alternatively a gravitational effect were predominant in the rotation axis of a galaxy), a higher concentration of objects near the rotational axis of the galaxy would be expected.

Section 2 presents the data and sample selection; the analysis and statistical tests are presented in Sects. 3 and 4, Sect. 5 discusses some details of the method to measure the anisotropy, and in Sect. 6 we interpret the results.

\section{Data and sample selection}

Two sources of public data have been used in this work:

- Third Reference Catalog of Bright Galaxies (de Vaucouleurs et al. 1991, updated on 16 February 1995; hereafter RC3): this catalogue is complete for galaxies with apparent diameters greater than 1 arcmin at the $D_{25}$ isophotal level and total $B$-band magnitudes $B_{T} \leq 15.5$, with a redshift $z \leq 0.05$. It contains a list of 23011 galaxies over the whole sky.

- Spectroscopic catalogue of the Third data release (DR3) of the Sloan Digital Sky Survey (Abazajian et al. 2005; hereafter SDSS): this catalogue covers 4188 square degrees and contains 51027 QSOs. The spectra have signal-to-noise ratios $S N R>4$ per pixel at $m_{\mathrm{g}}=20.2$. From these QSOs, we took the vetted subsample by Schneider et al. (2005) in which only those objects with $M_{i}<-22$ are selected (this implies that the low redshift QSOs are removed, and that the redshift range is from 0.08 to 5.41 ; their redshift is therefore always higher than those of the RC3 galaxies) and many of them have been checked manually to be QSOs (or otherwise removed). The total number of QSOs of this vetted catalogue is 46420 . We prefer to use the spectroscopic data instead of the photometric data used, for example, by Scranton et al. (2005) with a colour selection technique to separate the QSOs for reliable identification and an accurate estimate of the redshift. While we were working with these data, a fourth release of the survey was delivered that covered an area $14 \%$ larger (Adelman-McCarthy et al. 2006), but an updated vetted subsample has not been produced yet (and will not be produced until a fifth release of the data; D. P. Schneider, priv. comm.). In Sect. 4 we show some results of the unvetted 4 th release.

We are aware that the DR3 Quasar spectroscopic catalogue is not a statistical sample. Its QSOs do not all follow the same target selection, and the completeness depends on the positions, magnitudes, redshifts, etc., of the QSOs. Nonetheless, it is appropriate for the statistical analysis presented here because that completeness is related to the intrinsic properties of the QSOs and not to the orientation of nearby galaxies. Possible bias due to incompleteness and other systematic effects in the sample will be considered and quantified through Monte Carlo simulations in Sect. 4.

We take the RC3 catalogue and select all spiral galaxies with information on radial velocity $\left(v_{3 \mathrm{~K}}\right)$, the angular radius of the

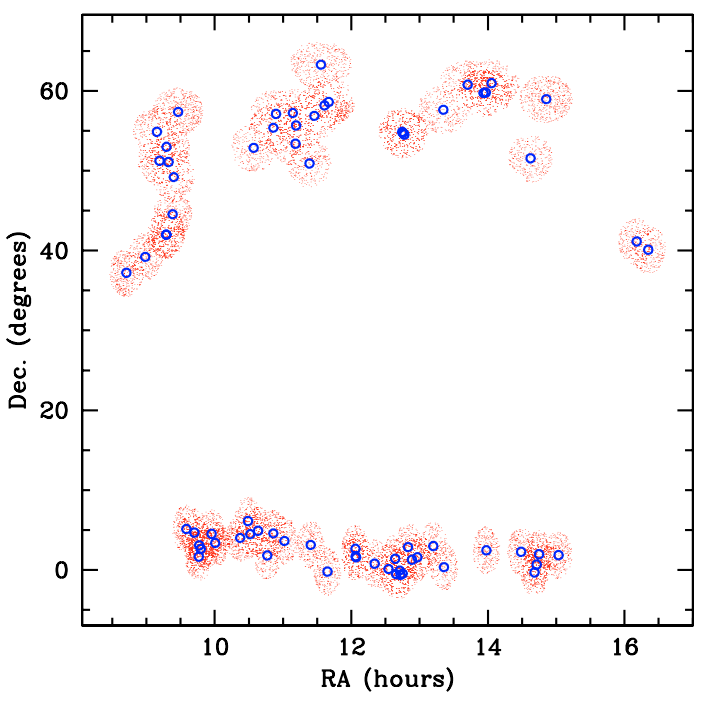

Fig. 1. Position on the sky of the sample of objects analysed in this article. Large open circles represent galaxies, and small dots QSOs. The plot corresponds to the case $\theta_{\max }=3^{\circ}$.

25th mag isophote $\left(\theta_{25}\right)$, the ratio between the major and minor axes and position angle, and whose coordinates are such that a circle of radius equal to $\theta_{\max }$ around each galaxy was totally covered by the 3rd release of the SDSS survey (see Sect. 5.3 for the use of circles partially covered). Spiral galaxies were chosen because the inclination of their discs is easily derived from the projected axial ratio $\left(\cos i=\frac{b}{a}\right)$. We reject the very nearby galaxies with $v_{3 \mathrm{~K}}<700 \mathrm{~km} \mathrm{~s}^{-1}$ (because their distances cannot be determined from their redshifts and because they would also extend too much in angular size with respect to the average sample; in any case, they are very few in number, and their inclusion would not significantly alter the statistics in this paper). We also select edge-on galaxies within the range $\pm 25^{\circ}(\sin i>0.906)$. There is no convenient a posteriori selection of galaxies: the selection of edge-on galaxies is necessary because they allow a clear separation between the rotation axis and the disc of the galaxy (the projection of the rotation axis is perpendicular to the disc of the galaxy and has a negligible component in the line of sight), and only spiral galaxies provide information for deriving the inclination to make possible the separation of edge-on galaxies. A range of 25 degrees $(i>65 \mathrm{deg})$ was taken in order to achieve a high enough number of galaxies within the nearly edge-on criterion ( $\sin i>0.906)$. If the limiting inclination were slightly larger or smaller, the statistical results given in this paper would be similar. If we selected only galaxies with an inclination very close to 90 degrees, we would have very few galaxies in our sample and the statistics would be much poorer; if we took the limiting inclination much lower than 65 degrees, we would introduce noise from nearly face-on galaxies.

We select the 44975 QSOs with $m_{\mathrm{g}} \leq 21$ within an angle of $\theta_{25}<\theta<\theta_{\max }$ from each galaxy (the lower limit in distance avoids possible effects of galactic extinction in background QSOs). There is no overlap in the redshift of the galaxies $(z<0.05)$ and QSOs $(z>\approx 0.08)$. Figure 1 shows the position of the resulting samples for the case $\theta_{\max }=3^{\circ}$.

\section{Analysis}

We compute the position angle of QSOs with respect to the minor axis of each galaxy. For $\theta_{\max }=3^{\circ}$ the total number of QSO-galaxy pairs is 25176 for $71 \mathrm{RC} 3$-galaxies. These 


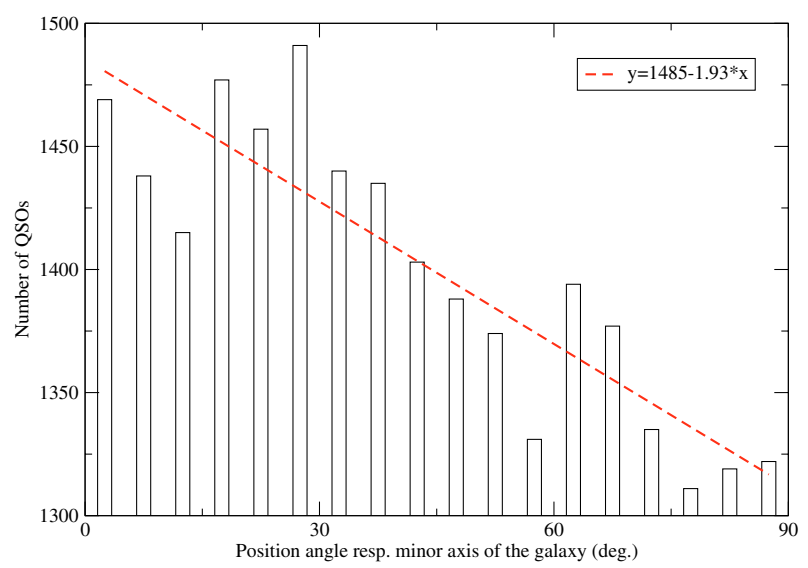

Fig. 2. Histogram representing the number of QSOs with $m_{\mathrm{g}}<21$ within a circle of radius equal to 3 degrees of $71 \mathrm{RC} 3$ galaxies as a function of position angle with respect to the minor axes of these galaxies.

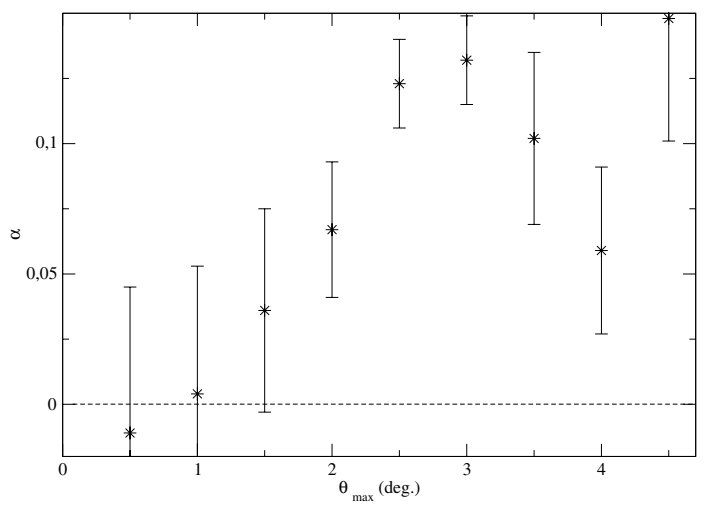

Fig. 3. $\alpha$ vs. $\theta_{\max }$ for QSOs with $m_{\mathrm{g}} \leq 21$ and any redshift (the points are not independent).

71 galaxies follow these constraints, with an average distance of $46 \mathrm{Mpc}$ (derived from $v_{3 \mathrm{~K}}$, assuming a Hubble constant of $72 \mathrm{~km} \mathrm{~s}^{-1} \mathrm{Mpc}^{-1}$ ) and a median distance of $32 \mathrm{Mpc}$. Some QSOs are counted more than once because they are within the radius $\theta_{\max }$ of two or more galaxies (see Fig. 1), but their relative position angles with respect to the different galaxies with which they are associated are uncorrelated, so they count as independent measures (see discussion in Sect. 5.2).

A histogram of all counts vs. relative position angle with respect to the minor axis of the galaxy is shown in Fig. 2 for $\theta_{\max }=3^{\circ}$. Clearly, there is a decrease in counts as we move from the minor to the major axis. The excess of counts in the minor axis direction is $\approx 13 \%$ higher than in the major axis direction. A fit for the counts per bin vs. position angle (PA) with a linear function

$C=C_{0}(1+\alpha r)$,

where $r=\left(1-\frac{\mathrm{PA}}{90^{\circ}}\right)$ (between 0 and 1$)$

gives $C_{0}=1311 \pm 13, \alpha=0.132 \pm 0.017$. This means a detection of anisotropy at the $7.8 \sigma$ level, although this is only the statistical significance of the survey, which does not represent the statistical significance of the real (unbiased) distribution of QSOs, as discussed in Sect. 4.

Other values of $\theta_{\max }$ (see Fig. 3) show the existence of anisotropy but the best significance is for $\theta_{\max } \approx 3^{\circ}$. For lower angles, there seems to be a lower value of $\alpha$, although for

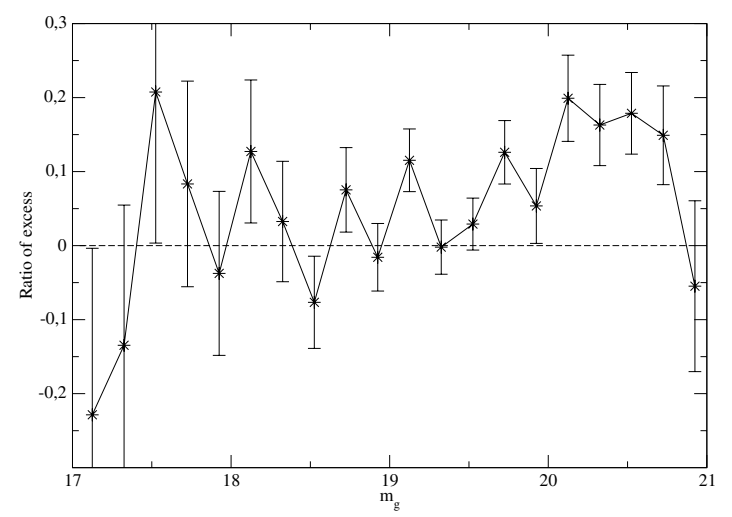

Fig. 4. Ratio of relative excess of QSOs in the range of position angles with respect to the minor axis $0-45^{\circ}$ over the range $45-90^{\circ}$ versus magnitude of QSOs.

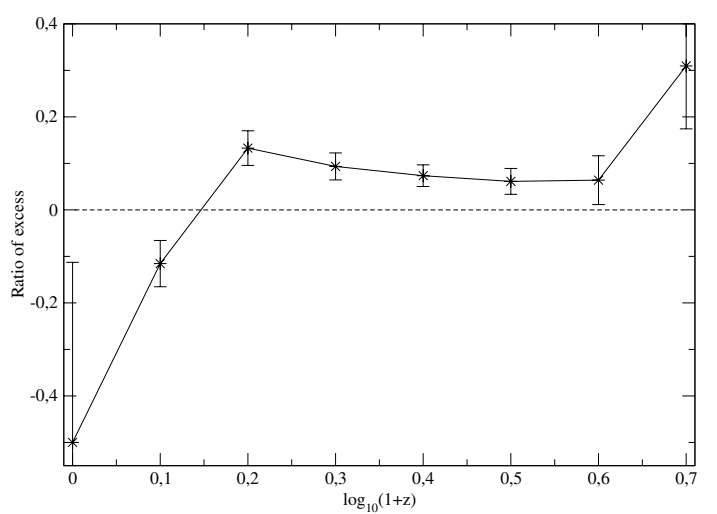

Fig. 5. Ratio of relative excess of QSOs in the range of position angles with respect to the minor axis $0-45^{\circ}$ over the range $45-90^{\circ}$ versus redshift of the QSOs.

$\theta_{\max }<1^{\circ}$ the number of QSOs is too low to draw any conclusions. For $\theta_{\max }>4^{\circ}$ the number of galaxies with total SDSS covered area in the circle with radius $\theta_{\max }$ is much lower (for $\theta_{\max }=4.5^{\circ}$ we have only 12 galaxies). In any case, the detection of $\alpha \neq 0$ (anisotropy) is clear, and the projected linear scale of around 1-1.5 Mpc (around 1.5-2.0 Mpc without projection) seems to have the highest ratio of anisotropy.

The excess in the range $0-45^{\circ}$ (13025 cases) over the range $45-90^{\circ}$ (12 151 cases) in Fig. 2 is $E_{45}=+874$ cases, a ratio of excess of $7 \%$. These extra 874 sources are preferably placed in the magnitude range $m_{\mathrm{g}}>19.4$ and the range $0.2<\log _{10}(1+z)<$ 0.5 (i.e. $0.6<z<2.2$ ), as can be observed in Figs. 4 and 5 . Curiously, the QSOs with $z<\approx 0.5$ significantly show the opposite trend on average: the excess is towards the major axis. The peaks with significant excess (higher signal/noise) or relative excess are more or less for $m_{\mathrm{g}} \sim 20.1$ and $z \sim 0.6$ respectively.

Figure 6 also shows how the QSOs with anisotropy are preferentially those with $m_{\mathrm{g}}>19.4$. The limit is near the maximum of the differential QSO counts; over 19.2 the counts are lower because of appreciable incompleteness (otherwise the differential counts should increase monotonically). One might suspect that the anisotropy has something to do with the incompleteness/bias of QSOs; however, it will be demonstrated in Sect. 4.1 that the main cause of the anisotropy is not that; at least the effects of incompleteness/bias, if there are any, are not enough to explain the observed anisotropy. Examining Fig. 6, one should also realize that there are two structures in the counts: two overlapping peaks, one with a maximum at $m_{\mathrm{g}} \approx 19.2$ and 


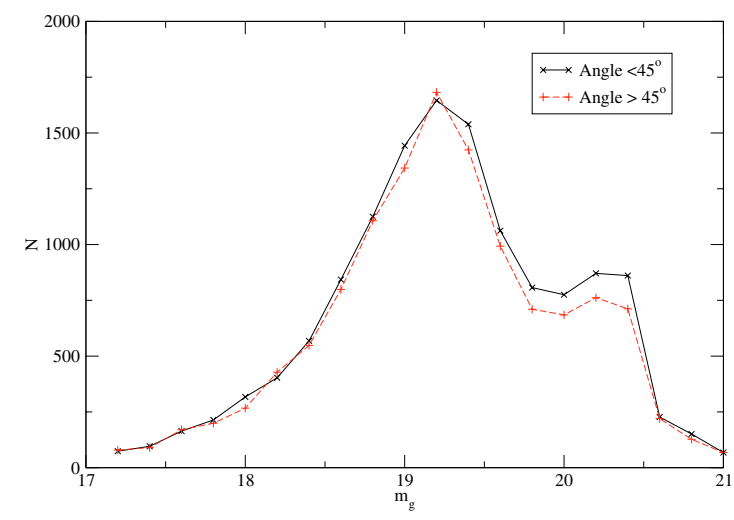

Fig. 6. Differential QSO counts in bins of 0.2 mag around the 71 galaxies that follow $\theta_{\max }=3^{\circ}$. The angle is with respect to the minor axis.

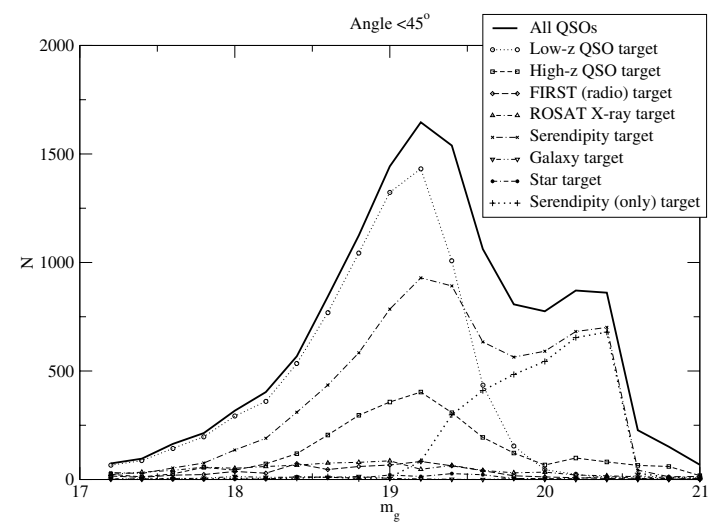

Fig. 7. Differential QSO counts in bins of 0.2 mag around the 71 galaxies which follow $\theta_{\max }=3^{\circ}$ with an angle respect to the minor axis of less than 45 degrees. Different SDSS samples attending to different criteria to preselect the targets are used.

another at $m_{\mathrm{g}} \approx 20.2$. Apparently, it is the second group of QSOs that is responsible for the anisotropy, and this is shown over $m_{\mathrm{g}}>19.4$ because this is the range where the number of QSOs in the second group is relatively significant. We suggest this to be the main reason for the manifestation of the anisotropy over $m_{\mathrm{g}}>19.4$ rather than the incompleteness. This second peak corresponds mainly to the criterion of target selection in the SDSS survey (Schneider et al. 2005) of serendipity sources with unusual colours; other targets do not make a such an important contribution (see Fig. 7). Values of $\alpha$ with the different target selections are given in Table 1 . When we take the QSOs that appear in the Serendipity target and exclude the other targets, we get a value of $\alpha=0.380 \pm 0.055$, an excess of $38 \%$ towards the minor axis instead of the average of $13 \%$ with the whole sample.

If we do a new analysis of the anisotropy with the added constraint that $m_{\mathrm{g}}>19.4,0.2<\log _{10}(1+z)<0.5$ (a total of 13308 cases), we get 7509 cases of QSO-galaxy association, and $\alpha$ to $0.313 \pm 0.042$. This represents more than a 30\% excess towards the minor axis with respect to the major axis. The analysis of the anisotropy only with the further added constraint that $m_{\mathrm{g}}>19.4$ gives 10204 cases of QSO-galaxy association with $\alpha=0.237 \pm 0.036$. If we restrict the analysis with the added constraint that $z>0.5(\log (1+z)>0.176)$ we get 22953 cases of QSO-galaxy association and $\alpha=0.156 \pm 0.017$. Although this simple constraint (in which we remove only $9 \%$ of the associations with respect to the analysis of the full sample) does
Table 1. Anisotropy of QSOs with different constraints.

\begin{tabular}{cccc}
\hline \hline$\theta_{\max }$ & Other constraints & Number of pairs & $\alpha$ \\
\hline $0.5^{\circ}$ & & 2807 & $-0.011 \pm 0.056$ \\
$1.0^{\circ}$ & & 8698 & $0.004 \pm 0.049$ \\
$1.5^{\circ}$ & & 14902 & $0.036 \pm 0.039$ \\
$2.0^{\circ}$ & 20562 & $0.067 \pm 0.026$ \\
$2.5^{\circ}$ & & 23819 & $0.123 \pm 0.017$ \\
$3^{\circ}$ & $m_{\mathrm{g}}>19.4$ & 25176 & $0.132 \pm 0.017$ \\
$3^{\circ}$ & $z<\log _{10}(1+z)<0.5$ & 10204 & $0.237 \pm 0.036$ \\
$3^{\circ}$ & $m_{\mathrm{g}}>19.4,0.2$ & 22953 & $0.313 \pm 0.042$ \\
$3^{\circ}$ & Low- $z$ QSOs target & 15434 & $0.064 \pm 0.026$ \\
$3^{\circ}$ & High- $z$ QSOs target & 5055 & $0.087 \pm 0.044$ \\
$3^{\circ}$ & FIRST (radio) target & 1184 & $0.203 \pm 0.085$ \\
$3^{\circ}$ & ROSAT (X-ray) target & 1728 & $0.031 \pm 0.087$ \\
$3^{\circ}$ & Serendipity target & 14445 & $0.179 \pm 0.030$ \\
$3^{\circ}$ & Serendipity (only) target & 5859 & $0.380 \pm 0.055$ \\
$3^{\circ}$ & Star target & 304 & $0.164 \pm 0.200$ \\
$3^{\circ}$ & Galaxy target & 205 & $-0.303 \pm 0.207$ \\
$3^{\circ}$ & & 21286 & $0.102 \pm 0.033$ \\
$3.5^{\circ}$ & & 14130 & $0.059 \pm 0.032$ \\
$4^{\circ}$ & & 9328 & $0.148 \pm 0.047$ \\
$4.5^{\circ}$ & & & \\
\hline
\end{tabular}

not give a much higher value of $\alpha$; it is much more significant statistically, as we comment in Sect. 4. The results of these and other analyses with different $\theta_{\max }$ are summarized in Table 1 .

The two-dimensional plot of the polar and radial distribution for two of the cases analysed are presented in Fig. 8. Apparently, QSOs are not randomly distributed around galaxies but show a quite significant trend to follow the minor axes of those galaxies. Since the median distance of the galaxies is $32 \mathrm{Mpc}$, the median linear projected maximum distance corresponding to $\theta_{\max }=3^{\circ}$ is $1.7 \mathrm{Mpc}$.

Figure 9 presents for the 71 galaxies considered in the case $\theta_{\max }=3^{\circ}$ the number of QSOs at $\pm 45^{\circ}$ from the minor and major axes respectively. The values are also given in Table 2 . There is in Fig. 9 a different degree of dispersion of anisotropies depending on the number of galaxies (galaxies with number less than 20 and higher than 60 present in general more dispersion in anisotropy). This may be due to the different levels of completeness for the different galaxies; the galaxies are numbered in Fig. 9 in right ascension order, so this most probably means that there are some regions of the sky in which the completeness is lower, and the number of background sources is also lower, as observed.

Two of the galaxies show a level of anisotropy higher than $3 \sigma$. The galaxy with highest anisotropy is PGC 36192 (NGC 3795). See the distribution of QSOs around it in Fig. 10. The excess $E_{45}$ (excess of QSOs of this galaxy in the region that are at position angles less than $45^{\circ}$ from the minor axis over those that have position angles larger than $45^{\circ}$ from it) is +99 (5.2 $\sigma$ over zero), and for this particular case $\alpha=2.02 \pm 0.28$, an excess of $200 \%$ towards the minor axis with respect to the major axis.

Among the 71 galaxies for $\theta_{\max }=3^{\circ}$, there are (according to the SIMBAD database, see Table 2): one LINER, two interacting galaxies, four low surface brightness galaxies, 15 galaxies in pairs/groups/clusters, and 49 normal galaxies. We looked for signs of correlation of the galaxy Hubble type with the anisotropy and found nothing. There is no correlation of $E_{45}$ with the position angle of the galaxy (see Table 2). There is some trend of higher anisotropy with lower distance (see Table 2): only $5( \pm 5) \%(1 / 19)$ of the galaxies with $d>50 \mathrm{Mpc}$ have $E_{45}>$ $2 \sigma\left(E_{45}\right)$, while for $27( \pm 7) \%(14 / 52)$ of galaxies $d<50 \mathrm{Mpc}$ with $E_{45}>2 \sigma\left(E_{45}\right)$. This may be because $3 \mathrm{deg}$ is too great a distance for galaxies with $d>50 \mathrm{Mpc}$ and the possible effect of 

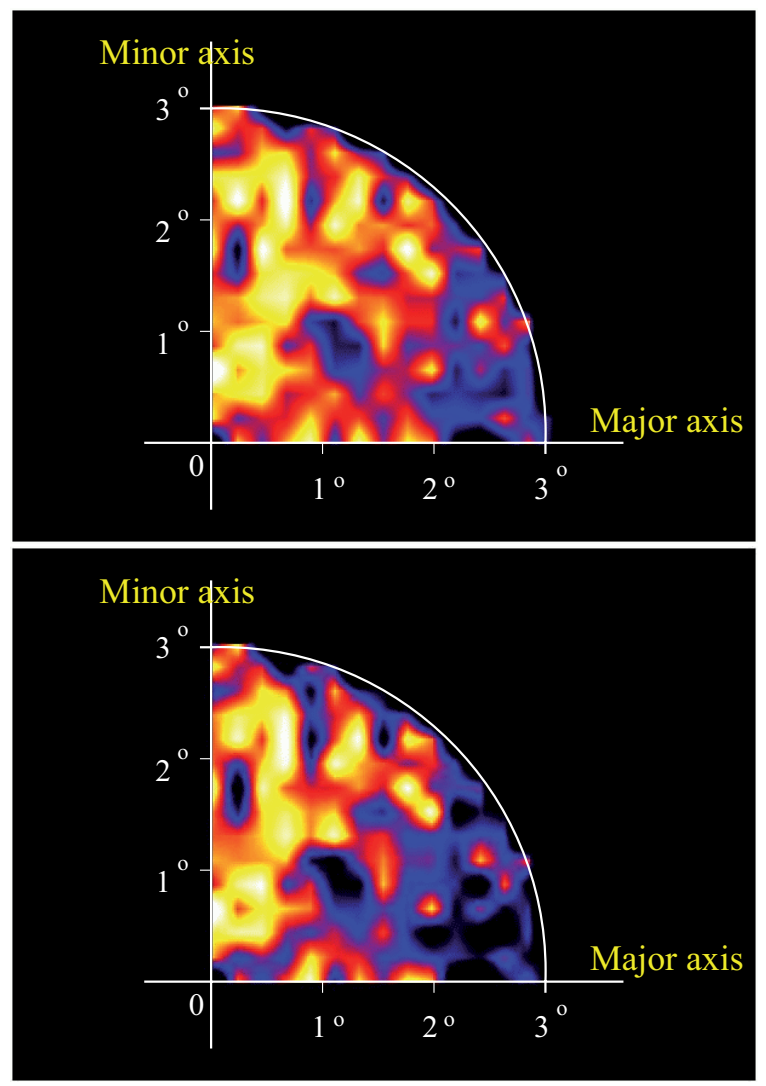

Fig. 8. Top: counts of QSOs (total: 25 176) as a function of position with respect to the minor/major axis angles of the corresponding 71 galaxies (see text). Counts were plotted in bins of $0.2^{\circ} \times 0.2^{\circ}$ (with an average of around 140 QSOs per bin) and smoothed/interpolated. The clearer colours indicate higher density. Note that towards the minor axis the average density of QSOs is greater than towards the major axis. Most of the fluctuations are presumably statistical (Poissonian). Bottom: the same but with the extra constraint $z>0.5$ (total: 22953 counts).

anisotropy is diluted in them. There also seems to be a tendency for interacting galaxies to have high anisotropy (see Table 2), but good statistics cannot be carried out with only two galaxies out of 71. And we have only one Seyfert (LINER), so neither can we comment on statistics of this type.

\section{Statistical significance of the anisotropy}

The spectroscopic survey SDSS is not complete for all redshifts up to the magnitude $m_{\mathrm{g}} \leq 21$. Moreover, we have observed that the completeness depends on position in the sky because the target selection varies from region to region. There are, for instance, important gradients of QSO density with the right ascension. Moreover, there are selection effects depending on the redshifts of the sources; SDSS is not equally capable of detecting sources at all redshifts, etc. Nonetheless, it is appropriate for our statistical analysis because that completeness has nothing to do with the orientation of nearby galaxies; that is, whether there are more or fewer QSOs has nothing to do with the presence of a galaxy some degrees away and even less with the orientation of the latter.

There will be a global effect in the dispersion from the isotropy due to the different gradients and systematics, which will be higher with respect to a perfect statistical survey, so we cannot trust the statistical error bars assuming a random distribution. For instance, the $7.8 \sigma$ level which we derived to be the
Table 2. Distribution of SDSS QSOs with $m_{\mathrm{g}} \leq 21$ within $\theta_{25}<\theta<3^{\circ}$ around the 71 selected spiral galaxies (with $i>65^{\circ}$ that have complete coverage of circles of radius $\theta_{\max }=3^{\circ}$ ). $E_{45}$ : excess of QSOs in the region which are at position angles less than $45^{\circ}$ from the minor axis over those that have position angles greater than $45^{\circ}$ from it (with Poissonian error). The galaxy type or environment is taken from the SIMBAD database classification. Distance $(d)$ derived from the redshift with a Hubble constant $72 \mathrm{~km} \mathrm{~s}^{-1} \mathrm{Mpc}^{-1}$. PA is the position angle of the major axis of the galaxy.

\begin{tabular}{|c|c|c|c|c|c|c|}
\hline$\overline{\# \#}$ & PGC-\# & Type/env. & $\overline{d(\mathrm{Mpc})}$ & $\overline{\overline{\mathrm{PA}}\left({ }^{\circ}\right)}$ & $\overline{\text { QSOs }}$ & $\overline{\overline{E_{45}}}$ \\
\hline 1 & 24453 & - & 54.6 & 72 & 427 & $+25( \pm 21)$ \\
\hline 2 & 25232 & - & 11.1 & 115 & 414 & $+58( \pm 20)$ \\
\hline 3 & 25781 & - & 35.8 & 120 & 369 & $-13( \pm 19)$ \\
\hline 4 & 25910 & Low S.Br. & 32.6 & 98 & 329 & $-15( \pm 18)$ \\
\hline 5 & 26232 & Interact. & 27.0 & 160 & 462 & $+58( \pm 21)$ \\
\hline 6 & 26238 & in Pair & 27.8 & 125 & 462 & $+44( \pm 21)$ \\
\hline 7 & 26246 & in Pair & 34.4 & 48 & 323 & $-25( \pm 18)$ \\
\hline 8 & 26351 & - & 9.9 & 140 & 307 & $+25( \pm 18)$ \\
\hline 9 & 26563 & - & 40.1 & 4 & 413 & $+37( \pm 20)$ \\
\hline 10 & 26631 & Interact. & 40.5 & 50 & 299 & $+49( \pm 17)$ \\
\hline 11 & 26856 & - & 46.6 & 123 & 548 & $-58( \pm 23)$ \\
\hline 12 & 27248 & - & 32.3 & 47 & 436 & $+50( \pm 21)$ \\
\hline 13 & 27734 & in Pair & 32.8 & 72 & 460 & $-16( \pm 21)$ \\
\hline 14 & 28010 & - & 30.6 & 127 & 463 & $-21( \pm 22)$ \\
\hline 15 & 28033 & in Group & 88.3 & 68 & 503 & $-41( \pm 22)$ \\
\hline 16 & 28148 & - & 30.7 & 15 & 505 & $+49( \pm 22)$ \\
\hline 17 & 28741 & - & 34.5 & 18 & 482 & $+64( \pm 22)$ \\
\hline 18 & 28939 & - & 33.1 & 151 & 529 & $+57( \pm 23)$ \\
\hline 19 & 30364 & - & 99.7 & 62 & 384 & $-36( \pm 20)$ \\
\hline 20 & 30885 & - & 54.3 & 111 & 299 & $+17( \pm 17)$ \\
\hline 21 & 31037 & - & 21.2 & 168 & 318 & $-2( \pm 18)$ \\
\hline 22 & 31269 & - & 101.3 & 156 & 323 & $-1( \pm 18)$ \\
\hline 23 & 31608 & - & 96.6 & 125 & 326 & $+14( \pm 18)$ \\
\hline 24 & 32153 & - & 18.6 & 159 & 313 & $+29( \pm 18)$ \\
\hline 25 & 32564 & - & 42.2 & 78 & 328 & $+32( \pm 18)$ \\
\hline 26 & 32570 & - & 19.3 & 130 & 335 & $+25( \pm 18)$ \\
\hline 27 & 32714 & in Group & 28.8 & 48 & 323 & $+1( \pm 18)$ \\
\hline 28 & 33234 & - & 20.7 & 20 & 307 & $+5( \pm 18)$ \\
\hline 29 & 33766 & - & 29.2 & 99 & 292 & $+16( \pm 17)$ \\
\hline 30 & 33964 & - & 42.3 & 38 & 297 & $+3( \pm 17)$ \\
\hline 31 & 34030 & - & 12.0 & 80 & 255 & $+19( \pm 16)$ \\
\hline 32 & 34971 & - & 13.9 & 142 & 312 & $+36( \pm 18)$ \\
\hline 33 & 35037 & - & 154.5 & 144 & 304 & $-12( \pm 17)$ \\
\hline 34 & 35249 & - & 25.3 & 128 & 300 & $+8( \pm 17)$ \\
\hline 35 & 35675 & - & 19.5 & 60 & 459 & $-13( \pm 21)$ \\
\hline 36 & 35900 & - & 19.2 & 9 & 351 & $+53( \pm 19)$ \\
\hline 37 & 36102 & - & 80.4 & 132 & 305 & $+19( \pm 17)$ \\
\hline 38 & 36192 & - & 17.3 & 53 & 373 & $+99( \pm 19)$ \\
\hline 39 & 38117 & in Cluster & 21.9 & 149 & 280 & $0( \pm 17)$ \\
\hline 40 & 38120 & in Cluster & 87.8 & 140 & 268 & $-14( \pm 16)$ \\
\hline 41 & 38188 & in Cluster & 89.4 & 100 & 263 & $-1( \pm 16)$ \\
\hline 42 & 39832 & Low S.Br. & 34.1 & 101 & 289 & $+1( \pm 17)$ \\
\hline 43 & 41618 & in Pair & 20.3 & 83 & 274 & $-28( \pm 17)$ \\
\hline 44 & 42255 & - & 76.5 & 148 & 278 & $+2( \pm 17)$ \\
\hline 45 & 42336 & - & 19.6 & 97 & 237 & $-7( \pm 15)$ \\
\hline 46 & 42689 & in Group & 28.5 & 63 & 254 & $+10( \pm 16)$ \\
\hline 47 & 42791 & in Group & 41.4 & 37 & 226 & $+32( \pm 15)$ \\
\hline 48 & 42942 & - & 71.7 & 177 & 309 & $+5( \pm 18)$ \\
\hline 49 & 42975 & LINER & 25.7 & 42 & 238 & $+30( \pm 15)$ \\
\hline 50 & 42998 & - & 69.0 & 97 & 307 & $+9( \pm 18)$ \\
\hline 51 & 43101 & - & 71.9 & 3 & 308 & $-16( \pm 18)$ \\
\hline 52 & 43397 & - & 20.6 & 0 & 286 & $+22( \pm 17)$ \\
\hline 53 & 43784 & - & 20.3 & 133 & 293 & $+7( \pm 17)$ \\
\hline 54 & 44392 & - & 21.4 & 89 & 326 & $+10( \pm 18)$ \\
\hline 55 & 45844 & - & 46.5 & 32 & 282 & $-13( \pm 17)$ \\
\hline 56 & 46589 & Low S.Br. & 31.4 & 153 & 335 & $+8( \pm 18)$ \\
\hline 57 & 46633 & - & 81.8 & 170 & 260 & $+2( \pm 16)$ \\
\hline 58 & 48534 & - & 32.1 & 30 & 466 & $+6( \pm 22)$ \\
\hline
\end{tabular}


Table 2. continued.

\begin{tabular}{ccccccc}
\hline \hline$\#$ & PGC-\# & Type/env. & $d(\mathrm{Mpc})$ & PA( $\left(^{\circ}\right)$ & QSOs & $E_{45}$ \\
\hline 59 & 49548 & - & 27.1 & 3 & 459 & $-17( \pm 21)$ \\
60 & 49712 & - & 43.4 & 167 & 470 & $-26( \pm 22)$ \\
61 & 49758 & - & 103.9 & 79 & 304 & $+6( \pm 17)$ \\
62 & 50069 & - & 30.3 & 174 & 489 & $+27( \pm 22)$ \\
63 & 51752 & - & 114.5 & 16 & 351 & $+85( \pm 19)$ \\
64 & 52266 & in Pair & 32.3 & 35 & 318 & $+24( \pm 18)$ \\
65 & 52455 & Low S.Br. & 27.3 & 107 & 388 & $0( \pm 19)$ \\
66 & 52558 & - & 28.1 & 66 & 427 & $-19( \pm 21)$ \\
67 & 52665 & in Pair & 27.0 & 170 & 446 & $+44( \pm 21)$ \\
68 & 53043 & - & 30.8 & 144 & 546 & $+1( \pm 23)$ \\
69 & 53683 & - & 20.0 & 115 & 374 & $+10( \pm 19)$ \\
70 & 57386 & in Cluster & 135.0 & 173 & 298 & $+22( \pm 17)$ \\
71 & 57882 & in Cluster & 141.9 & 65 & 292 & $-24( \pm 17)$ \\
\hline All & - & - & - & - & 25176 & $+874( \pm 159)$ \\
\hline \multicolumn{7}{c}{-}
\end{tabular}

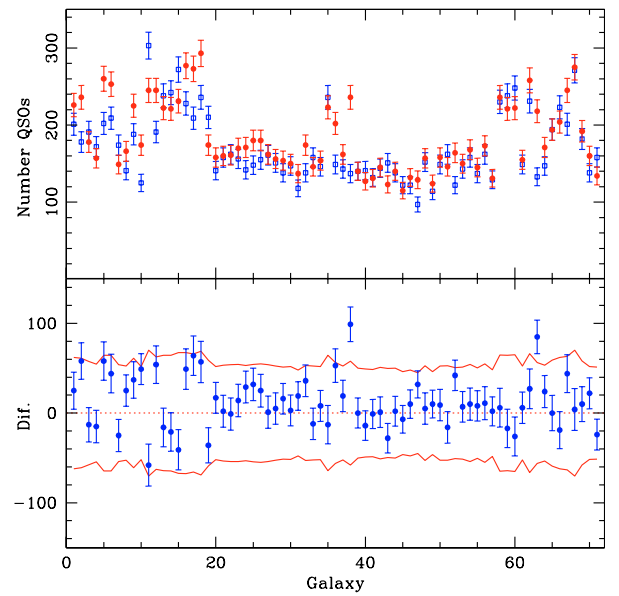

Fig. 9. Top: Number of QSOs at $\pm 45^{\circ}$ from the minor axis (open points), and from the major axis (filled points) around the minor and major axes for the 71 galaxies ordered in right ascension considered in the case $\theta_{\max }=3^{\circ}$. Bottom: difference between number of QSOS around the minor and major axes. The continuum lines represent the $\pm 3 \sigma$ level.

significance of non-zero slope of the fit of expression (1) applied to Fig. 2 would indicate the level of anisotropy of the Schneider et al. (2005) sample, which is both due to the anisotropy in the real distribution and to the systematic deviations of a homogeneous sample. We can also calculate this level of significance through Kolmogorov-Smirnov test applied to the same data, which also gives a low probability of being compatible with an angle-independent distribution: $P_{K-S}=2 \times 10^{-8}$ (equivalent to $5.6 \sigma$ in a Gaussian distribution). Or simply we can count in the same data the excess in the range $0-45^{\circ}$ (13025 cases) over the range $45-90^{\circ}$ (12151 cases) in Fig. $2: E_{45}=+874$ cases (Poissonian error \pm 159 ), i.e. $5.5 \sigma$. In order to distinguish which part of the significance of the anisotropy is due to the real distribution, we have to carry out Monte Carlo simulations.

\subsection{Monte Carlo simulations with random position angle of the galaxies}

Given the present distribution of QSOs in Schneider et al. (2005), we have randomly generated the position angles of 71 galaxies in the RC3 (the correlation of position angles, if any, are small and insignificant; see Sect. 4.4), and we have measured the same numbers $P_{K-S}$ and $E_{45}$. We have carried 50000 Monte Carlo simulations of this type. The distribution of $E_{45}$ for the
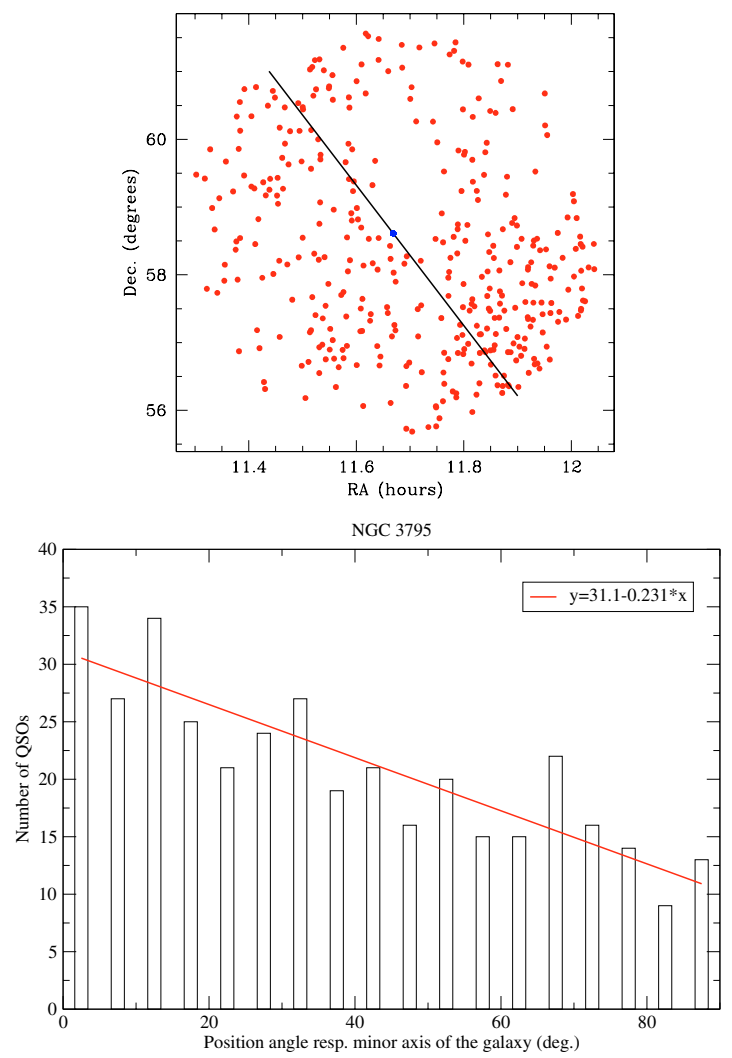

Fig. 10. Top: distribution of QSOs around NGC 3795. The line represents the minor axis direction. Bottom: histogram representing the number of QSOs as a function of position angle with respect to the minor axis of NGC 3795 within 3 deg of it. A constant dependence is excluded within the $7.2 \sigma$ level.

case without constraints is shown in Fig. 11. This distribution results in a Gaussian distribution with $\sigma \approx 250$. Our detection of anisotropy would be at the $3.5 \sigma$ level. Table 3 presents the results and the statistical significance for this and other cases with further constraints.

It is remarkable that merely by removing the cases with $z<$ 0.5 (only $9 \%$ of the cases) we increase the statistical significance considerably (to $3.9 \sigma$ ). This is due to the negative signal of the anisotropy for these removed cases, as shown in Fig. 5. As said, this better signal/noise of the anisotropy is also observed when comparing the two cases shown in Fig. 8.

\subsection{The same test rejecting the three farthest outliers}

We repeat the statistical analysis rejecting the three farthest outliers identified in Table 2 and Fig. 9. By definition, outliers do not behave like the others, either because of the physics or because of certain uncontrolled factors; separating outliers might be a test to better understand the properties of the general population, and of the outliers themselves if they are physically different. We repeat the case only with the constraint $\theta_{\max }=3^{\circ}$.

We now remove three galaxies from Table 2: PGC 28741, PGC 36192 and PGC 51752, all of them with the highest positive value of $E_{45}$, so it is expected that we are going to reduce the signal of the anisotropy. The number of galaxies is now 68 . The number of pairs is $23970 . E_{45}=632, \alpha=0.097 \pm 0.018$, $P_{K-S}=4 \times 10^{-5}$. The Monte Carlo simulations on the random orientations of the galaxies give a significance $\sigma\left(P_{K-S}\right)=2.7$, $\sigma\left(E_{45}\right)=2$.6. It seems that there is nothing wrong with the previous statistics. The signal is still there in spite of the removal 
Table 3. $\sigma$ from the results of Monte Carlo simulations with random orientations of the galaxies for the measured values of $P_{K-S}$ and $\left|E_{45}\right|$ in the real distribution.

\begin{tabular}{cccccc}
\hline \hline Constraint & $N_{\text {pairs }}$ & $P_{K-S}$ & $\sigma\left(P_{K-S}\right)$ & $E_{45}$ & $\sigma\left(\left|E_{45}\right|\right)$ \\
\hline$\theta_{\max }=3^{\circ}$ & 25176 & $2 \times 10^{-8}$ & 3.5 & 874 & 3.5 \\
$\theta_{\max }=3^{\circ}, m_{\mathrm{g}}>19.4,0.2<\log _{10}(1+z)<0.5$ & 7509 & $5 \times 10^{-12}$ & 3.3 & 601 & 3.3 \\
$\theta_{\max }=3^{\circ}, m_{\mathrm{g}}>19.4$ & 10204 & $4 \times 10^{-10}$ & 3.2 & 644 & 3.4 \\
$\theta_{\max }=3^{\circ}, z>0.5$ & 22953 & $8 \times 10^{-10}$ & 3.9 & 919 & 3.9 \\
\hline
\end{tabular}

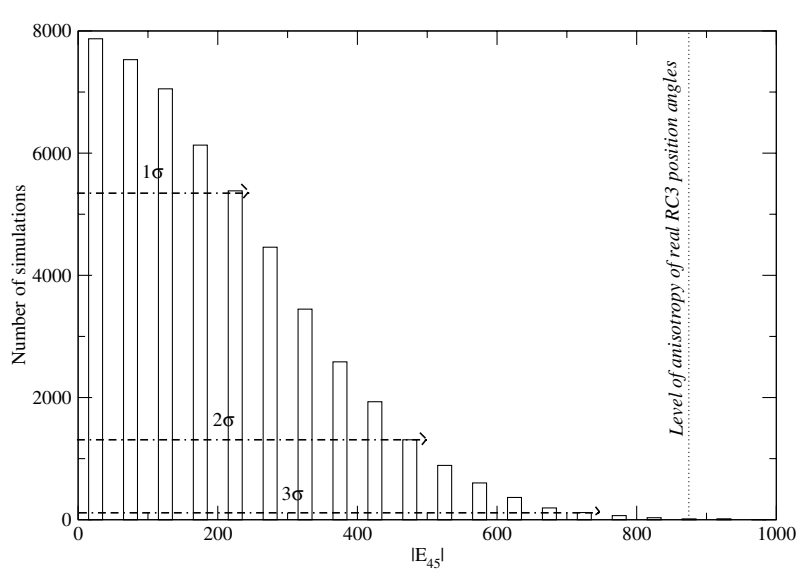

Fig. 11. Results of the $\left|E_{45}\right|$, excess in the range $0-45^{\circ}$ over the range $45-90^{\circ}$ (with a total of 25176 galaxy-QSO associations), of a Monte Carlo simulation with random position angles for the RC3 galaxies. As shown, the real position angles give a significant anisotropy of $E_{45}=$ 874 , which is the $3.5 \sigma$ level.

of the three farthest outliers. It is lower than $3.5 \sigma$ and is reduced to 2.6 or $2.7 \sigma$, but this is precisely what would be expected if we remove members that are known to have the highest anisotropy. There is an average trend for a positive $\alpha$ that is not due to the presence of a single galaxy (or two or three) with some special circumstances.

\subsection{Tests with subsamples}

We now perform the same calculations with subsamples containing $90 \%$ of the identified QSO-galaxy pairs. If the sample is pure, the anisotropy will decrease slightly owing to the smaller number of objects. If outliers have a strong effect, most of the simulations will have a slightly higher anisotropy and a few of them will have a much lower anisotropy (when the outliers are randomly rejected).

For this test with 1000 different subsamples (each subsample with $90 \%$ randomly selected pairs from the initial 25176 in 71 galaxies), we calculate the quantity $E_{45} / N_{\text {pairs }}$, which gives a measure of the anisotropy. This time we do not calculate the significance for each subsample, because this is very timeconsuming; in any case, the significance does not need to be calculated for each different subsample because it is more or less proportional to $E_{45} / N_{\text {pairs }}$. The results of these 1000 subsamples are shown in Fig. 12. The median value of $E_{45} / N_{\text {pairs }}$ in these 1000 subsamples is 0.0347 , the same value that was obtained with the original total sample of 25176 pairs. Nothing anomalous is present in this distribution of values of $E_{45} / N_{\text {pairs }}$ so we do not think that a few outliers are producing the anisotropy but that it is a characteristic of the average distribution. All 1000 subsamples show a relative excess of QSOs with position angle less than 45 degrees of $E_{45} / N$ over 0.025 .

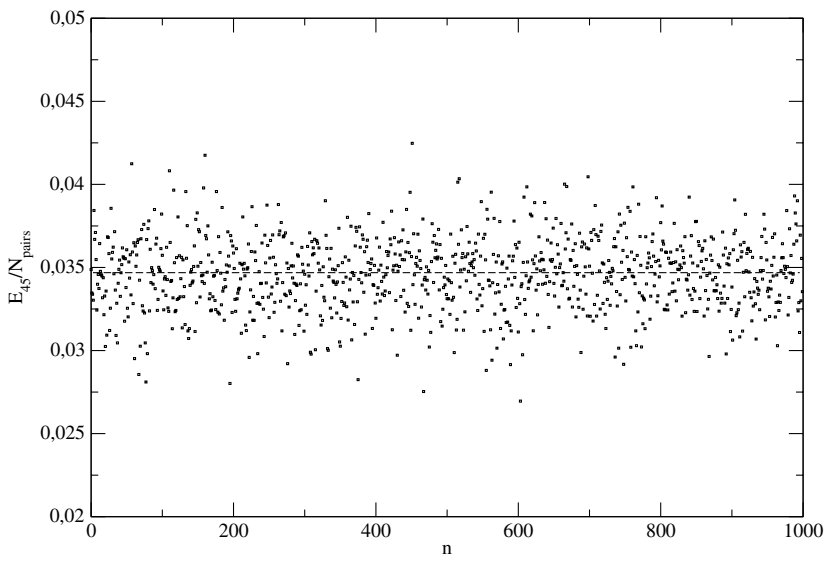

Fig. 12. Distribution of values of the relative excess of QSOs with position angle less than 45 degrees for 1000 subsamples ( $n$ from 1 to 1000), where each subsample has $90 \%$ randomly selected pairs from the initial 25176 in 71 galaxies. The dashed line represents the value for the original 25176 pairs: $E_{45} / N_{\text {pairs }}=0.0347$.

\subsection{Monte Carlo simulations with random QSO distributions}

In the previous subsection, we showed that, given the actual distribution of SDSS QSOs, a random distribution of position angles in the RC3 galaxies should not show the level of anisotropy found; that is, the anisotropy is not due to the peculiarities of the QSO distribution itself. Now from the position and orientation of the $71 \mathrm{RC} 3$ galaxies we generate a random Poissonian and homogeneous distribution of background sources through a Monte Carlo simulation and we shall see whether the observed level of anisotropy is attained. These should serve to test that the 71 RC3 galaxies have no significant bias responsible for the anisotropy. In fact, we have also checked that the distribution of position angles in our sample (see Table 2) is compatible with being randomly distributed (according to the KolmogorovSmirnov test: a probability of $83 \%$ for the hypothesis of null correlation). There is no significant correlation of position angles, although, of course, some small correlation within the noise cannot be rejected; in any case, this small correlation would be negligible for our statistical purposes.

The results indicate that the probability of obtaining an anisotropy of the measured values is roughly equal to the probability given by the Kolmogorov test. From 873 simulations, we got $89.9 \%$ with $P_{K-S}>0.1,9.5 \%$ with $0.01<P_{K-S}<0.1$ and $0.5 \%$ with $0.001<P_{K-S}<0.01$; none with $P_{K-S}<0.001$. This distribution is to be expected if the probabilities obey the Kolmogorov-Smirnov distribution. These results indicate that the probability of getting the observed value $P_{K-S}=2 \times 10^{-8}$ from a random distribution of QSOs is negligible. This means that there is no selection effect in the RC3 sample. 


\subsection{Tests with other catalogues than Schneider et al. (2005)}

We have also carried out the same test with the spectroscopic galaxies of the 3rd SDSS release up to $m_{\mathrm{g}}=21$ (333 314 galaxies), instead of the QSO sample, and we have found that those galaxies with a difference of velocity with respect to RC3 galaxies higher than $3000 \mathrm{~km} \mathrm{~s}^{-1}$ (to avoid their being associated with the main RC3 galaxy) have an insufficiently significant level of anisotropy. As always, the RC3 galaxies are spiral and nearly edge-on (inclination greater than 65 degrees). The result gives $\alpha=-0.029 \pm 0.010$ (more galaxies towards the major than towards the minor axis), $P_{K-S}=2 \times 10^{-4}$, and the Monte Carlo simulations (with random orientation of the RC3-galaxies) show that this has a probability of $38 \%$ (equivalent to only $0.9 \sigma$ ).

If instead of Schneider et al.'s (2005) vetted QSO catalogue we use the complete SDSS 3rd data release for QSOs (we also put the constraint that the difference in velocity of the QSO and galaxy must be higher than $3000 \mathrm{~km} \mathrm{~s}^{-1}$ ), the anisotropy is detected with $\alpha=0.111 \pm 0.022, P_{K-S}=3 \times 10^{-6}$, and, according to Monte Carlo simulations, the anisotropy has a level of $2.9 \sigma$. The reason for the lower significance of the anisotropy (with the Schneider et al. sample it was $3.5 \sigma$ ) is that, apart from further spurious contamination, it includes QSOs with $M_{i}>-22$, which are mostly of low redshift, and this has negative $\alpha$, as shown in Fig. 5, which reduces the average signal. With the added constraint $z>0.5, \alpha=0.154 \pm 0.024, P_{K-S}=2 \times 10^{-9}$ and the Monte Carlo simulations indicate anisotropy at a level of $3.6 \sigma$.

We can also use the newest release, SDSS 4th (DR4, Adelman-McCarthy et al. 2006), which covers 4783 sq. degrees and contains 61049 QSOs with $m_{\mathrm{g}}<21.0$. Although the area covered is only $14 \%$ greater than that covered by the DR3, the total number of RC3 galaxies that have circles of radius 3 degrees totally covered is somewhat higher: 127 instead of 71, and the number of QSO-galaxy associations (some QSOs may have more than one association with a galaxy) is 47271 instead 25 176. This improves the statistics slightly. However, since the vetted catalogue has not yet been produced and will not be produced until the 5th release is delivered (Schneider, priv. comm.) only the analysis of the anisotropy of the complete SDSS 4th data release is possible, as in the previous paragraph. The result is: $\alpha=0.073 \pm 0.013, P_{K-S}=2 \times 10^{-5}$, and according to Monte Carlo simulations the anisotropy has a level of $2.5 \sigma$. With the extra constraint $z>0.5, \alpha=0.106 \pm 0.012, P_{K-S}=7 \times 10^{-9}$ and the Monte Carlo simulations suggest anisotropy at a level $3.3 \sigma$. The average anisotropy is somewhat lower than with only DR3 sources, possibly the first 71 galaxies have on average a higher anisotropy; in any case, the differences are roughly within the expected random fluctuations from one sample to the other.

Another search for anisotropy was made using the $2 \mathrm{dF}$ QSO spectroscopic catalogue (Croom et al. 2004) with $m_{\mathrm{B}}<21$ (23660 QSOs in total). This catalogue has the disadvantage of a much lower coverage, around 700 square degrees, and its two strips have a width of 5 degrees, which does not allow complete circles of radius 3 degrees (we consider the full areas of the two $75 \mathrm{deg} \times 5 \mathrm{deg}$ ). We do the analysis with nearly edge-on spiral RC3 galaxies that have at least $80 \%$ of the circles with $\theta_{\max }=3^{\circ}$ covered (26 galaxies) and we normalize the QSO counts by dividing by the covered area. This results in poorer statistics than SDSS, but it is illustrative that we also observe the anisotropy, although with very low significance. Figure 13 shows a histogram of densities: $\alpha=0.089 \pm 0.022, E_{45}=1.6 \pm 0.5 \mathrm{deg}^{-2}$; a probability according to Monte Carlo simulations of $51 \%(0.7 \sigma)$, which is too insignificant to be considered a detection of anisotropy but is compatible with the results from the SDSS survey. If instead

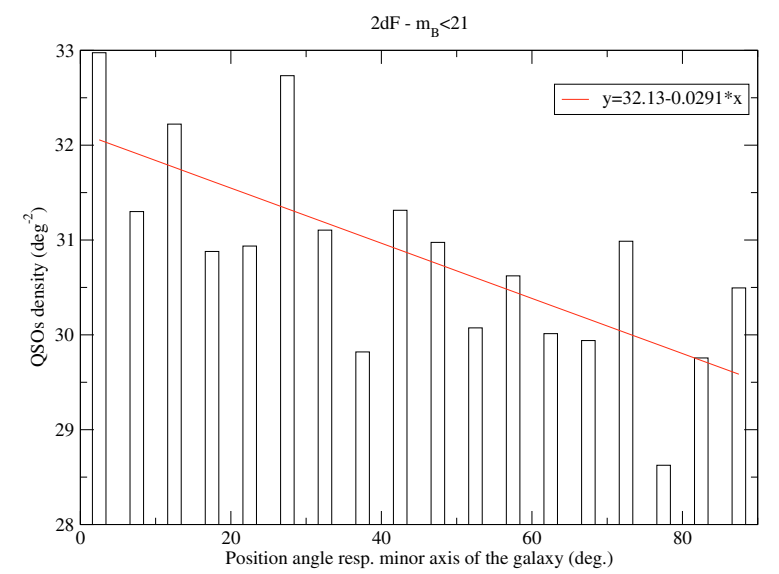

Fig. 13. Histogram representing the $2 \mathrm{dF}$ QSO density with $m_{\mathrm{B}}<21$ within a circle of radius equal to 3 degrees of $26 \mathrm{RC} 3$ galaxies (more than $80 \%$ of the circle covered) as a function of position angle with respect to the minor axes of these galaxies.

of $80 \%$ minimum coverage, we use only galaxies with $90 \%$ minimum coverage, we have 13 galaxies, $\alpha=0.109 \pm 0.028, E_{45}=$ $2.1 \pm 0.6 \mathrm{deg}^{-2}$; and a probability according to Monte Carlo simulations of $22 \%(1.2 \sigma)$, still insignificant. For $\theta_{\max }=2.5^{\circ}$, and a coverage greater than 90\%: 20 galaxies, $\alpha=0.084 \pm 0.017$, $E_{45}=1.2 \pm 0.6 \mathrm{deg}^{-2}$; and a probability according to Monte Carlo simulations of $34 \%(1.0 \sigma)$, insignificant.

\section{Some considerations towards measuring the anisotropy}

\subsection{Anisotropy as a function of linear distance}

One possible question about our measure is why we counted the QSOs within a fixed angular distance (3 degrees) instead of a fixed linear distance. Since we know the distance of the RC3 galaxies, we could count the QSOs within a linear projected distance. Indeed, we tried to measure the anisotropy in such a way and we also detected the anisotropy but the statistical significance was not as high as $3.5 \sigma\left(3.9 \sigma\right.$ for $\left.z_{\text {QSO }}>0.5\right)$. With identical criteria, using Schneider et al.'s (2005) QSO catalogues but for the maximum linear distance of $1.8 \mathrm{Mpc}$ (where we got the best values of the anisotropy) instead of 3 degrees of angular distance, the results are: 113 RC3-galaxies, 16572 galaxyQSO associations, $\alpha=0.138 \pm 0.038, P_{K-S}=2 \times 10^{-5}$, with a significance according to Monte Carlo simulations of $2.6 \sigma$. The two-dimensional distribution as a function of distance is plotted in Fig. 14. For $z_{\mathrm{QSO}}>0.5$, there are 15079 galaxy/QSO associations, $\alpha=0.172 \pm 0.042, P_{K-S}=2 \times 10^{-7}$, with a significance according to Monte Carlo simulations of $3.1 \sigma$.

This slightly lower significance of the anisotropy may be due to the following reasons: i) the selected number of very nearby galaxies $(\sim 10 \mathrm{Mpc})$ with the linear distance criterion is lower than the angular distance criterion because most of them do not follow the constraint of being totally covered by the SDSS 3rd release survey, a galaxy at $\sim 10 \mathrm{Mpc}$ would give a circle with radius $\sim 9$ degrees, which is very unlikely to be totally covered by the SDSS-3rd release survey (and, as said in Sect. 3, there is a slight trend towards higher anisotropy for lower distances); ii) the number of QSOs per galaxy is lower for more distant galaxies, because they cover a lower area. These arguments can explain the numbers we obtained. 


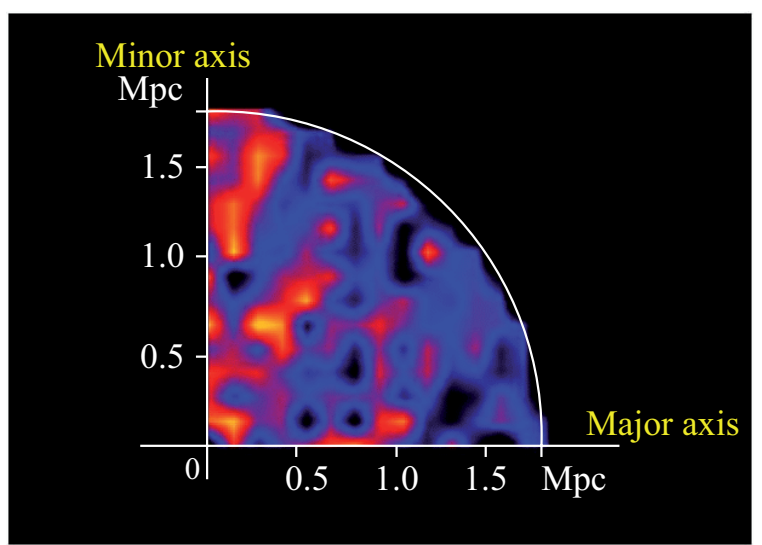

Fig. 14. Counts of QSOs (total: 16572) as a function of position with respect to the minor/major axis angles of the corresponding 113 galaxies for linear distances lower than $1.8 \mathrm{Mpc}$ (see text). Counts were plotted in bins of $0.12 \mathrm{Mpc} \times 0.12 \mathrm{Mpc}$ (average around 110 QSOs per bin) and smoothed/interpolated. The clearest colours indicate higher density.

One might restrict the range of distances to avoid this selection effect in some measure. For instance, if we take only the RC3 galaxies within the range of distance $20<d<40 \mathrm{Mpc}$ (around the median value of $32 \mathrm{Mpc}$; i.e. $0.005<z_{\text {gal }}<0.010$ instead of $\left.z_{\text {gal }}<0.050\right)$. We would then have $20 \mathrm{RC} 3$ galaxies, 9457 QSO-galaxy associations , $P_{K-S}=2 \times 10^{-7}$, Monte Carlo significance: $2.8-\sigma$. For $z_{\mathrm{QSO}}>0.5$ : $8631 \mathrm{QSO}-$ galaxy associations, $P_{K-S}=1 \times 10^{-9}$, Monte Carlo significance: $3.3 \sigma$. The signal/noise is a little better with this restriction but still less than the values obtained with the condition $\theta<3^{\circ}$. Here, the reason is possibly the lower number of galaxy-QSO associations used for the statistics. Whatever the reason, it seems that the condition $\theta<3^{\circ}$ gets higher anisotropy than linear separation $<1.8 \mathrm{Mpc}$.

\subsection{Counting some QSOs twice or more times}

An interesting question is how to select QSO/galaxy pairs. At present, we have selected all the QSOs around a given RC3 galaxy; consequently, if two galaxies are closer together than 6 degrees, they will have some common QSOs within the circle of radius 3 degrees; some QSOs count twice or more in the QSO/galaxy association. Since the position and orientation of the galaxies are random, the different counts with the same QSO should be independent. Even in the case that they were not independent, the Monte Carlo simulations of Sect. 4.1 would include the possible factors due to it, and the significance derived therein would contain them. We have estimated the anisotropies with other criteria in which a QSO counts only once: associating it with the closest/farthest galaxy in the case that two or more galaxies include it within their circles, and the observed anisotropy has only slight differences: $E_{45} / N_{\text {pair }}=$ $0.0328 / 0.0373$ respectively for closest/farthest criterion instead of 0.0347 (Table 3 ). This is explicable perhaps because we would be adding a selection effect in which the QSOs are on average closer or farther from the galaxies; or by coordinates, in which a QSO is associated with the first/last galaxy in the list of RC3, which is ordered by coordinates, and in this case we found more notable differences, which we attribute to the gradient of QSO density in right ascension present in SDSS survey. Summing up, the most objective criterion is to include all QSOs around a galaxy even if they are counted twice or more; other criteria can introduce extra bias; moreover, we have fewer counts and
Table 4. Statistics with different minimum allowed coverages of the circles with $\theta_{\max }=3^{\circ}$.

\begin{tabular}{cccccc}
\hline \hline Min. cover. & $N_{\text {gal }}$ & $N_{\text {pairs }}$ & $\alpha$ & $E_{45}\left(\mathrm{deg}^{-2}\right)$ & $\sigma\left(\left|E_{45}\right|\right)$ \\
\hline $100 \%$ & 71 & 25176 & $0.132 \pm 0.017$ & 0.872 & 3.5 \\
$99 \%$ & 117 & 41888 & $0.124 \pm 0.015$ & 0.791 & 4.0 \\
$98 \%$ & 135 & 48131 & $0.133 \pm 0.013$ & 0.837 & 4.4 \\
$98 \%\left(z_{Q}>0.5\right)$ & 135 & 43839 & $0.158 \pm 0.014$ & 0.903 & 4.8 \\
$97 \%$ & 143 & 50938 & $0.105 \pm 0.013$ & 0.677 & 3.8 \\
$95 \%$ & 162 & 57000 & $0.084 \pm 0.010$ & 0.546 & 2.9 \\
$90 \%$ & 188 & 64791 & $0.067 \pm 0.011$ & 0.452 & 2.5 \\
\hline
\end{tabular}

consequently less significance in the detection of the anisotropy, although whatever the criteria, the Monte Carlo simulations will include them and the significance will be correctly calculated.

\subsection{With incomplete circles}

So far, we have used circles that are covered completely by the SDSS survey (except for $2 \mathrm{dF}$ data). We have performed other measures with circles partially covered, normalizing the number of counts with the area covered per segment, and we have observed that: i) it is a trivial result that the number of galaxies is higher for lower minimum allowed coverages; ii) lower minimum coverages in the circles produce higher dispersion and lower average anisotropy in our case. When the non-covered holes are larger, random effects dominate (perhaps because we are taking border areas with significant gradients of completeness; also, the errors in the determination of the areas of the segments might contribute) and in our case the average random anisotropy is fortuitously negative. We have perfomed measures only with galaxies of low coverage and we have tested that they produce a negative $\alpha$, in which Monte Carlo simulations show that it is not significant. When we mix low-coverage with fullcoverage circles we are wrecking/smoothing the effect observed with the full-coverage circles alone.

The best compromise between points i) and ii) to get the highest significance of the anisotropy was found for the constraint of $98 \%$ as minimum allowed coverage. This gives 135 galaxies (instead of 71 galaxies with total coverage), 48131 QSO-galaxy pairs , $\alpha=0.133 \pm 0.013, E_{45}=0.837 \mathrm{deg}^{-2}$ whose significance according to Monte Carlo simulations is $4.4 \sigma$ (a probability of 1 in 90000 ). For $z>0.5$ the significance according to Monte Carlo simulations is $4.8 \sigma$ (a probability of 1 in $600000)$. Table 4 gives further values for other coverages.

\section{Interpretation}

This distribution of objects around galaxies is similar to that found by Zaritsky et al. (1997) and Azzaro et al. (2006) (the Holmberg effect), i.e. a higher density of objects in the minor axis direction. However, their distribution was for satellite galaxies associated with the main galaxy and our objects are QSOs with very different redshifts with respect to the main galaxy. The projected scales are also different $\sim 300-500 \mathrm{kpc}$ for the satellites, and $\sim 1-1.5 \mathrm{Mpc}$ in our case. The Holmberg effect has been explained as a consequence of the preferential capture by the parent galaxy of satellites orbiting near the equatorial plane. From a purely phenomenological point of view, the effect discovered here is similar to the Holmberg effect. Although we have 


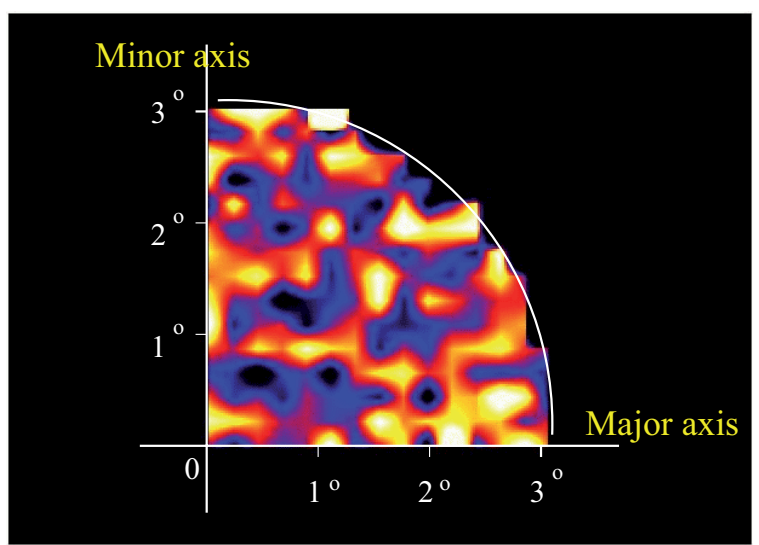

Fig. 15. Distribution of the average colour $\langle(g-r)\rangle$ as a function of position with respect to the minor/major axis angles of the corresponding 71 galaxies (see text). Average: 0.173 . Bins of $0.2^{\circ} \times 0.2^{\circ}$, smoothed/interpolated. The clearest colour indicate reddest colour.

not tried to build a detailed model, we have considered three possibilities:

- Extinction: It might be that there is some extinction along the major axis of the galaxies that is high enough to reduce significantly the number of QSOs observed in that direction. The affected range of redshifts $(z>0.5)$ contain QSOs in which $\mathrm{H}_{\alpha}$ lines cannot be detected in the optical SDSS survey (and Ly- $\alpha$ cannot be observed for $z<2.2$ ), so lines like $\mathrm{H}_{\alpha}$ (or Ly- $\alpha$ ) are not used for their identification as QSOs when $0.5<z<2.2$; instead, other, fainter lines are used, and they will be near the limit of detection at $m_{\mathrm{g}}$ around 20 . An excess of extinction along the major axis of $\sim 0.1 \mathrm{mag}$ could produce a selection effect in the SDSS survey that removes some of the objects to be classified as QSOs because the signal/noise of some lines becomes lower than the limit of detection. For example, if we have a QSO whose equivalent width of the most intense line has got a signal/noise of 4 without extinction, with extinction this signal/noise would be lower than 4, so the line of this object would not be catalogued and therefore this object would not be classified as a QSO. However, it is difficult to accept that some clouds along the major axis associated with a galaxy are as far as 1.5-2.0 Mpc (see Fig. 14) from it. Intergalactic clouds might reside at $1.5-2 \mathrm{Mpc}$ from a galaxy but there is no reason in principle to think they are in the plane of the galactic disc rather than in a random distribution. A random distribution of intergalactic clouds would not produce anisotropy. Moreover, we have computed the polar and radial distribution of $g-r$ colours of our sample of QSOs and we find no appreciable differences between the mean colour of objects at angles $\leq \geq \pm 45^{\circ}$ from the minor axis of the galaxy (Fig. 15 presents the twodimensional distribution of such colours; the average colour as a function of the position angle from the minor axis is $\left.\langle(g-r)\rangle=0.1727 \pm 0.0022+1.9 \pm 4.3 \times 10^{-5} P A(\mathrm{deg})\right)$, so unless a grey-dust extinction is present, this explanation does not seem appropriate.

- Gravitational lensing: As said in the introduction, previous detection of a correlation between high redshift QSOs and low redshift galaxies has been tentatively attributed to effects of gravitational lensing in the halo of foreground galaxies. This solution does not work in general (Zhu et al. 1997;
Tang \& Zhang 2005), and it also has important problems in explaining the phenomenon of this paper:

- The dependence of the anisotropy on the redshift of the QSOs (excess over $z>0.5$ and defect over $z<0.5$ ) cannot be explained in terms of the distance of the source and the lens. All gravitational lens effects, whatever the model, have an Einstein radius $\theta_{E}$ such that $\theta_{E}^{n}$ is proportional to $\frac{D_{\mathrm{ls}}}{D_{\mathrm{s}}}$ (e.g. $n=2$ for microlensing, $n=1$ for singular isothermal sphere approximation), with $D_{\mathrm{ls}}$ and $D_{\mathrm{s}}$ respectively the distance lens-source, source-observer. $\frac{D_{\mathrm{ls}}}{D_{\mathrm{s}}}$ is nearly one for all QSOs at $z>0.08$ (assuming a distance of the galaxy of $\left.32 \mathrm{Mpc}, \frac{D_{\mathrm{ls}}}{D_{\mathrm{s}}}>0.9\right)$. We cannot understand with this hypothesis the absence of anisotropy for most QSOs with $z<0.5$. The dependence on the magnitude shown in Fig. 6 is also difficult to understand. we cannot understand why there is no lensing effect for the brightest QSOs.

- The linear scales of the effect discovered here seem too large for the size of the halos as estimated in $N$-body CDM or $\Lambda \mathrm{CDM}$ simulations. For instance, typical virial radii are $\sim 100-300 \mathrm{kpc}$ in these models. Although Prada et al. (2005) have demonstrated that actual halos extends at least up to $2-3 R_{\text {vir }}$, the density at this distances is so low that it seems difficult to produce significant lensing effects. A point to note is that such simulations predict elongated halos (Allgood et al. 2006; Mandelbaum et al. 2006), whose major axes in the inner parts tend to be orthogonal to the galactic discs, while in the outer parts the halos tend to be randomly orientated. The anisotropy found in this work would need elongated structures perpendicular to the galactic discs and extending out to $\sim 1.5 \mathrm{Mpc}$ (see Fig. 14).

- The amount of mass necessary is huge. Assuming as a first approximation that the increase in counts in SDSS is due to an increase in the magnitudes of the background sources (this is not exactly true, because the magnitudes of an object refer to the average flux of the object in a passband, while the SDSS detection criteria depend on the signal/noise of some lines, but the order of magnitude should not be very different), the average enhancement is $\langle q\rangle \approx 1.07$ (an increase of $7 \%$ in the counts) in the counts over $\approx 14 \mathrm{deg}^{2}$ (the region within $\theta<3^{\circ}$ and polar angle within $45^{\circ}$ from the minor axis). The magnification factor $\mu$ due to gravitational lensing (see, for instance, Eq. (1) of Zhu et al. 1997) is related to $q$ by means of

$$
q=\frac{N\left(m_{\mathrm{b}}+2.5 \log _{10} \mu\right)}{N\left(m_{\mathrm{b}}\right)} \frac{1}{\mu}
$$

where $N$ is the cumulative QSO counts up to magnitude $m_{\mathrm{b}}$. With the Boyle et al. (2000) counts $(N=1981-$ $214.2 m_{\mathrm{b}}+5.792 m_{\mathrm{b}}^{2}$ (López-Corredoira \& Gutiérrez 2004 , Appendix A); in the real distribution of QSOs, not in our sample which is incomplete), $\langle\mu\rangle \approx 1.28$ for $m_{\mathrm{b}}=20.0$ on average over $\approx 14 \mathrm{deg}^{2}$. For $m_{\mathrm{b}}=19.5$, it is $\langle\mu\rangle \approx 1.08$ and for $m_{\mathrm{b}}=20.5$ there is no value of $\mu$ that gives an enhancement as high as $q=1.07$. Let us consider the average value of $m_{\mathrm{b}} \approx 20.0:\langle\mu\rangle \approx 1.28$. With a single isothermal sphere approximation $\mu \approx \frac{\theta}{\theta-\theta_{\mathrm{E}}}$, where $\theta_{\mathrm{E}}$ is the Einstein radius; whose average over a circle of radius $\theta_{\max }>>\theta_{\mathrm{E}}$ is $\langle\mu\rangle \approx 1+\frac{2 \theta_{\mathrm{E}}}{\theta_{\max }}$. Therefore, in our case it would be $\theta_{\mathrm{E}} \approx 1100$ arcsec, which would require an excess of mass in the minor axis region with respect to the major axis region of a whole rich cluster of galaxies (for the typical galaxy distance of $32 \mathrm{Mpc}$ ) in a single spiral galaxy (Wu 1996). Even if the mass is distributed over 
many lenses, the total necessary mass is of the same order because $\langle(\mu-1)\rangle \propto \sum_{i} \theta_{\mathrm{E}, i} \propto \sum_{i} M_{i}=M_{\text {total }}$, although the numbers can change depending on the size of the lenses. With a point-like microlensing approach (Paczyński 1986; Wu 1996), $\mu \approx \frac{u^{2}+2}{u \sqrt{u^{2}+4}}, u=\theta / \theta_{\mathrm{E}}$, whose average over a circle of radius $\theta_{\max } \gg \theta_{\mathrm{E}}$ is $\langle\mu\rangle \approx 1+\frac{2 \theta_{\mathrm{E}}^{2}}{\theta_{\max }^{2}}$, it results in $\theta_{\mathrm{E}}=2900$ arc seconds. In the case of microlensing, $\theta_{\mathrm{E}}^{2}$ is proportional to the mass $\left(\theta_{\mathrm{E}}^{2}=\frac{4 G M}{d c^{2}}\right.$ for distances of the QSOs much greater than the distance of the galaxy; Paczyński 1986; Wu 1996; Tang \& Zhang 2005), $\langle(\mu-1)\rangle \propto \sum_{i} \theta_{\mathrm{E}, i}^{2} \propto \sum_{i} M_{i}=M_{\mathrm{total}}$, so again it does not matter whether the mass is distributed in one or many sources: the average magnification will be the same. In our case, we would need a mass $M \sim 4 \times 10^{16} M_{\odot}$ for $d=32 \mathrm{Mpc}$, an impossible value.

- Non-cosmological redshifts: At least some QSOs are associated with nearby parent galaxies. They would have absolute magnitudes between -10 and -14 in $g$. In this unorthodox scenario, QSOs might be ejected with velocity enough to become gravitationally unbounded to the galaxy. Arp (1998a, Figs. 3-27) proposes ejection along the minor axis, although out to distances of $0.5 \mathrm{Mpc}$. If we were to assume this hypothesis to be correct, why do we find this effect at distances up to $2-3^{\circ}$ ? Maybe because our sample have relatively faint QSOs, and QSOs with small angular separation from a galaxy have higher luminosities on average (Dravskikh \& Dravskikh 1996) that correspond to lower apparent magnitudes. Possibly the same effect of anisotropy would be observed for bright QSOs on scales less than $1^{\circ}$. Bright QSO excess might not be detected because the number of such QSOs in SDSS is very low for their excess to be detected statistically. Why do the QSOs with redshift $z>0.5$ preferably show this anisotropy and not other redshifts? If Arp's hypothesis of ejection were right, maybe QSOs with lower redshifts are further away or turned into galaxies, but this places the maximum distance even beyond $\approx 1.5 \mathrm{Mpc}$, so the problem with distance in Arp's hypothesis still remains. That interacting galaxies appear among the galaxies with the highest anisotropy in the distribution might point some relation between both phenomena. Therefore, Arp's hypothesis qualitatively predicts the observed anisotropy but it fails in the distance estimation of the QSOs from the galaxies by a factor of $\sim 3$.

Could the detected anisotropy be a statistical fluctuation? It might be, but it is a very low probability one: for instance $3.5 \sigma$ means a probability $5 \times 10^{-4}$ (with no preselection of QSOs and galaxies except the angle $\theta_{\max }=3^{\circ}$ ). Moreover, Fig. 5 shows a clear dependence on redshift which is not expected for a random sample of QSOs (there is a clear non-random trend of anisotropy depending on $z$ ), so the significance increases to $3.9 \sigma$ only by removing the QSOs with $z<0.5$ (9\% of the total). Is this possibly due to chance? We think that this is not very likely, but that is precisely the nub of the question. Here, we deliver this new challenge in the long-running puzzle of this old topic: the relationship between high redshift QSOs and nearby galaxies. In any case, since this is the first time that this anisotropy has been observed and the possibility of a chance fluctuation, although unlikely, is not totally discarded, we prefer at present to be prudent and just say that it is a "tentative" detection that should be corroborated by other groups before any extraordinary claims are made.
Acknowledgements. Thanks are given to the anonymous referee, Halton C. Arp (MPIA, Garching, Germany), Ruben J. Díaz (Córdoba, Argentina) and D. P. Schneider (Pennsylvania, USA) for useful comments, and T. J. Mahoney (IAC, Tenerife, Spain) for proof-reading of the text. We acknowledge use of RC3 catalogue its authors. Funding for the creation and distribution of the SDSS Archive has been provided by the Alfred P. Sloan Foundation, the Participating Institutions, the National Aeronautics and Space Administration, the National Science Foundation, the US Department of Energy, the Japanese Monbukagakusho, and the Max Planck Society. The SDSS Web site is http://www.sdss.org/. The SDSS is managed by the Astrophysical Research Consortium (ARC) for the Participating Institutions. The Participating Institutions are The University of Chicago, Fermilab, the Institute for Advanced Study, the Japan Participation Group, The Johns Hopkins University, the Korean Scientist Group, Los Alamos National Laboratory, the Max-Planck-Institute for Astronomy (MPIA), the Max-Planck-Institute for Astrophysics (MPA), New Mexico State University, University of Pittsburgh, University of Portsmouth, Princeton University, the United States Naval Observatory, and the University of Washington. The 2dF QSO Redshift Survey (2QZ) was compiled by the 2QZ survey team from observations made with the 2-degree Field on the Anglo-Australian Telescope. The authors were supported by the Ramón y Cajal Programme of the Spanish Science Ministry.

\section{References}

Abazajian, K., Adelman-McCarthy, J. K., Agüeros, M. A., et al. 2005, AJ, 129, 1755

Adelman-McCarthy, J. K., Agüeros, M. A., Allam, S. S., et al. 2006, ApJS, 162, 38

Allgood, B., Flores, R. A., Primack, J. R., et al. 2006, MNRAS, 367, 1781

Arp, H. C. 1966, Science, 151, 1214

Arp, H. C. 1967 , ApJ, 148, 321

Arp, H. C. 1998a, Seeing Red, Apeiron, Montreal

Arp, H. C. 1998b, ApJ, 496, 661

Arp, H. C. 1999a, Active Galactic Nuclei and Related Phenomena, ed. Y.

Terzian, E. Khachikian, \& D. Weedman, Astronomical Society of the

Pacific, S. Francisco, 347

Arp, H. C. 1999b, A\&A, 341, L5

Arp, H. C., \& Hazard, C. 1980, ApJ, 240, 726

Arp, H. C., \& Russell, D. 2001, ApJ, 549, 802

Azzaro, M., Patiri, S. G., Prada, F., \& Zentner, A. R. 2006,

[arXiv:astro-ph/0607139]

Benítez, N., Sanz, J. L., \& Martínez-González, E. 2001, MNRAS, 320, 241

Boyle, B. J., Shanks, T., Croom, S. M., et al. 2000, MNRAS, 317, 1014

Burbidge, G. R. 1999, in Cosmological Parameters and the Evolution of the Universe, ed. K. Sato (Dordrecht: Kluwer), 286

Burbidge, G. R. 2001, PASP, 113, 899

Burbidge, E. M., Burbidge, G. R., Solomon, P. M., \& Strittmatter, P. 1971, ApJ, 170,223

Chu, Y., Zhu, X., Burbidge, G., \& Hewitt, A. 1984, A\&A, 138, 408

Croom, S. M., Smith, R. J., Boyle, B. J., et al. 2004, MNRAS, 349, 1397

de Vaucouleurs, G., de Vaucouleurs, A., Corwin, H. G., et al. 1991, Third Reference Catalog of Bright Galaxies (New York: Springer, RC3)

Dravskikh, A. F., \& Dravskikh, Z. V. 1996, Astron Zh., 73, 19. Translated into english in 1996, Astron. Rep., 40, 13

Gaztañaga, E. 2003, ApJ, 589, 82

Guimaraes, A. C. C. 2005, [arXiv: astro-ph/0510719]

Kovner, I. 1989, ApJ, 341, L1

López-Corredoira, M., \& Gutiérrez, C. M. 2004, A\&A, 421, 407

Mandelbaum, R., Hirata, C. M., Broderick, T., Seljak, U., \& Brinkmann, J. 2006, MNRAS, 370, 1008

Narlikar, J. V., \& Das, P. K. 1980, ApJ, 240, 401

Nollenberg, J. G., \& Williams, L. R. 2005, ApJ, 634, 793

Paczyński, B. 1986, ApJ, 304, 1

Prada, F., Klypin, A. A., Simonneau, E., et al. 2006, ApJ, 645, 1001 Richards, G. T., Nichol, R. C., Gray, A. G., et al. 2004, ApJS, 155, 257 Schneider, D. P., Hall, P. B., Richards, G. T., et al. 2005, AJ, 130, 367 Scranton, R., Ménard, B., Richards, G. T., et al. 2005, ApJ, 633, 589 Tang, S. M., \& Zhang, S. N. 2005, Chin. J. Astron. Astrophys., 5, 147 Wu, X.-P. 1996, Fund. Cosmic Phys., 17, 1

Zaritsky, D., Smith, R., Frenk, C. S., \& White, S. D. M. 1997, ApJ, 478, L53 Zhu, Z.-H., Wu, X.-P., \& Fang, L.-Z. 1997, ApJ, 490, 31 